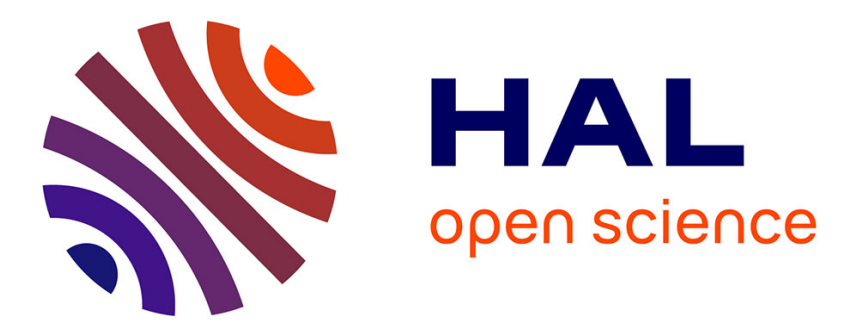

\title{
Study on color space for the performance of degraded face image recognition
}

Xinwei Liu, Christophe Charrier, Marius Pedersen, Patrick Bours

\section{To cite this version:}

Xinwei Liu, Christophe Charrier, Marius Pedersen, Patrick Bours. Study on color space for the performance of degraded face image recognition. Media Watermarking, Security, and Forensics Conference, IS\&T Electonic Imaging 2018, Jan 2018, Burlingame, United States. hal-01709097

\section{HAL Id: hal-01709097 https://hal.science/hal-01709097}

Submitted on 14 Feb 2018

HAL is a multi-disciplinary open access archive for the deposit and dissemination of scientific research documents, whether they are published or not. The documents may come from teaching and research institutions in France or abroad, or from public or private research centers.
L'archive ouverte pluridisciplinaire $\mathbf{H A L}$, est destinée au dépôt et à la diffusion de documents scientifiques de niveau recherche, publiés ou non, émanant des établissements d'enseignement et de recherche français ou étrangers, des laboratoires publics ou privés. 


\title{
Study on color space for the performance of degraded face image recognition
}

\author{
Xinwei Liu ${ }^{1,2}$, Christophe Charrier ${ }^{1}$, Marius Pedersen ${ }^{2}$, and Patrick Bours ${ }^{2}$ \\ ${ }^{1}$ Normandie Univ, UNICAEN, ENSICAEN, CNRS, GREYC, Caen, France \\ ${ }^{2}$ NTNU - Norwegian University of Science and Technology, Gjøvik, Norway
}

\begin{abstract}
Color provides important information and features for face recognition. Different color spaces possess different characteristics and are suitable for different applications. In this paper, we propose to investigate how different color space components influence the performance of degraded face recognition. Towards this goal, nine color space components are selected for the evaluation. In addition, four different types of image-based degradations are applied to face image samples in order to discover the impact on the performance of face recognition. The experimental results show that, all selected color components have similar influence to the performance of face recognition system depend on the acquisition devices and the experimental setups.
\end{abstract}

\section{Introduction}

Biometrics are more and more popular in recent years. Among all the existing biometric modalities, face recognition is one of the well known technologies. Thanks to the development of color imaging technology and biometric recognition application, face images captured under unconstrained environment by classical devices such as smartphone, webcams or low-cost camera can be used for face recognition [1]. However, many of existing face recognition approaches are only using grayscale images converted from RGB images by taking the average of the three color components. The drawback of this method is that we do not consider the impact of each color component in a RGB image, and, moreover, we ignore the influence of other color spaces than the RGB space. Back to 1999, color information has been noticed due to its importance in face recognition [2]. Unlike grayscale images, color face images are represented in the most commonly used RGB color space. By including color information in face recognition, the unique characteristics that are exclusive to color face image have become relevant to recognition performance [1]. The impact of color components on recognition performance lead us to further analyze the representation of color information. We can investigate through various color spaces to explore additional information from the color image that can be used to increase recognition performance. Another factor that influence the performance of face recognition is the quality of face sample images. The color face images can be captured under unconstrained environment conditions. Therefore, some image-based degradations could be introduced during acquisition process. Using such face images for recognition is a more challenging issue [1]. In order to investigate how the above mentioned two factors affect the performance of face recognition, the goal of this paper is to determine whether an optimal color space component give better performance than a traditional grayscale image on distorted face samples. This paper is organized as follows: we first present state-of-the-art concerning the studies of color space and sample quality for face recognition. Then, we introduce the experiment setup and illustrate the experimental results. We conclude this work at the end.

\section{State-of-the-art}

There are many researches using color face image for recognition. Choi et al. [3] proposed to use: color local Gabor wavelets and color local binary pattern for the purpose of face recognition. Jones and Abbott [4] explored the extraction of features from color face images by using an extended hypercomplex Gabor filtering method. Wang et al. [5] represented a color face recognition approach based on two-dimensional principal component analysis. Yang and Liu [6] presented a general discriminant model for color face recognition. Choi et al. [7] proposed a metric called 'variation ratio gain', which is aimed to prove theoretically the significance of color effect on low-resolution faces within wellknown subspace face recognition frameworks. It quantitatively characterizes how color features affect the recognition performance with respect to changes in face resolution. They also conducted performance evaluation studies to show the effectiveness of color on low-resolution faces. However, the number of studies that investigate the influence of color information on face recognition is limited. Yip and Sinha [8] suggested that color cues do play a role in face recognition and their contribution becomes evident when shape cues are degraded. Under such conditions, recognition performance with color images is significantly better than that with grayscale images. Their experimental results indicated that the contribution of color may lie not so much in providing diagnostic cues to identity as in aiding low-level image-analysis processes such as segmentation. Torres et al. [2] stated that a common feature found in practically all technical approaches proposed for face recognition is the use of only the luminance information associated to the face image. It is necessary to know if this is due to the low importance of the color information in face recognition or due to other less technical reasons. They performed a variety of tests using a global eigen approach, which was modified to cope with the color information. Their results show that the use of the color information embedded in an eigen approach can improve the recognition rate when compared to the same scheme which uses only the luminance information. Yoo et al. [9] presented color processing for face recognition systems and the results showed that color information helps the performance of face recognition and found that specifically $\mathrm{YCbCr}$ and $\mathrm{YCg}^{\prime} \mathrm{Cr}$ ' color spaces are the most appropriate for face recognition. Bours and Helkala [10] investigated whether using one of the three color lay- 
ers of the RGB image could give better recognition performance compared to the greyscale converted image. Finally, Hemery et al. [11] studied different color spaces for representing an image for the face authentication application. They used a generic algorithm based on a matching of key points using SIFT descriptors computed on one color component. Ten color spaces have been studied on four large and significant benchmark databases. The results showed that all color spaces do not provide the same efficiency and the use of the color information allows an interesting improvement of verification results.

Face recognition is still a challenging issue when degraded face images are acquired [12]. It has been proved that face sample quality has significant impact on accuracy of biometric recognition [13]. Low sample quality is a main reason for matching errors in biometric systems and may be the main weakness of some applications [13]. Recently, several standardizations on biometric sample quality have been finalized, especially for face modality: ISO/IEC JTC 1/SC 37 19794-5 Information technology - Biometrics - Biometric data interchange formats - Part 5: Face image data [14] and ISO/IEC TR 29794-5 Information technology - Biometric sample quality - Part 5: Face image data [15]. In the standard [14], requirements for the face image data record format as well as the instruction of photographing high quality face images are presented. Some important aspects should be considered in order to meet the basic face image quality requirements: pose angle, facial expression, visibility of pupils and irises, focus, illumination and so on. In the standard [15], definition and specification of methodologies for computation of objective, quantitative quality scores for facial images are proposed. The face image quality is given by the relation to the use of facial images with face biometric systems [15]. The use of low quality face images in face recognition affects the performance of the system. There are many factors that can affect face image quality, and the performance of biometric systems. It is important to take into account image quality attributes that influence face quality. Both imagebased and modality-based face quality attributes are presented in [15]. According to the standard [15], we can find the following image-based face quality attributes: 1) image resolution and size; 2) noise; 3) illumination intensity; 4) image brightness; 5) image contrast; 6) focus, blur and sharpness; and 7) color.

\section{Experimental setup}

\section{Color face image database - $G C^{2}$ Multi-modality Biometric Database}

If we want to investigate how image-based distortions affect the performance of face recognition by taking into account color spaces, it is recommended to use a database without modalitybased distortions [16, 17]. Most of the existing color face databases contain both image-based and modality-based distortions. The ' $\mathrm{GC}^{2}$ Multi-modality Biometric Database' is a new database without modality-based distortions. For the color face sub-dataset in this database, three cameras were used to capture face images: 1) a Lytro [18] first generation Light Field Camera (LFC) (11 Megapixels), 2) a Google Nexus 5 embedded camera (8 Megapixels), and 3) a Canon D700 with Canon EF 100mm $\mathrm{f} / 2.8 \mathrm{~L}$ Macro Lens (18 Megapixels). There are 50 subjects in the dataset and 15 samples images are taken for each subject. Totally, 2250 face images are obtained in the face dataset.
In order to obtain image-based distortions, we follow the protocol introduced in [16, 17]: four image-based attributes are used to degrade face images for all color space components. We used Matlab R2016a to conduct the experiment as following:

- Contrast distortions. There are two kinds of contrast distortions: too low and too high contrast. We use Matlab function 'J $=$ imad just $\left(I,\left[\right.\right.$ low $_{\text {in }} ;$ high $\left._{\text {in }}\right],\left[\right.$ low $_{\text {out }} ;$ high $\left.\left._{\text {out }}\right]\right)$ ', which maps the values in $I$ (original face image) to new values in $J$ (degraded face image) such that values between $l o w_{\text {in }}$ and high $h_{\text {in }}$ map to values between low out and high $h_{\text {out }}$. For low contrast, the $l o w_{\text {in }}$ and $h i g h_{\text {in }}$ values are set to 0 and 0.4 low $_{\text {out }}$ and high $h_{\text {out }}$ values are set to 0 and 1 . For high contrast, the $l o w_{\text {in }}$ and $h i g h_{\text {in }}$ values are set to 0.6 and 1, low out $_{\text {and }}$ high $h_{\text {out }}$ values are set to 0 and 1 .

- Sharpness distortions. We generate two sharpness distortions: motion blur and Gaussian blur. For motion blur we use Matlab function ' $h=f$ special ('motion', len, theta)', which returns a filter to the linear motion of a camera by len pixels, with an angle of theta degrees in a counterclockwise direction. The len value is set to 30 and the theta is set to 45 . For Gaussian blur we use function ' $h=$ fspecial ('gaussian', hsize, sigma)', which returns a rotationally symmetric Gaussian lowpass filter of size hsize with standard deviation sigma (positive). The hsize value is set to [25 25] and the sigma is set to 2 .

- Luminance distortion. There are two kinds of luminance distortions: too low and too high luminance. We use Matlab function ' $J=\operatorname{imadjust}\left(I,\left[\right.\right.$ low $_{\text {in }} ;$ high $\left._{\text {in }}\right],\left[\right.$ low $_{\text {out }} ;$ high $\left.\left._{\text {out }}\right]\right)$ ', again to simulate luminance distortions. For low luminance, the $l o w_{\text {in }}$ and $h i g h_{\text {in }}$ values are set to 0 and 1, low out and high $h_{\text {out }}$ values are set to 0 and 0.2 . For high luminance, the $l o w_{\text {in }}$ and $h i g h_{\text {in }}$ values are set to 0 and $1, l o w_{\text {out }}$ and $h i g h_{\text {out }}$ values are set to 0.8 and 1 .

- Artifacts. We introduce two artifacts to face images: poisson noise and JPEG compression artifacts. We use Matlab function ' $J=$ imnoise $\left(I,{ }^{\prime}\right.$ poisson $\left.{ }^{\prime}\right)$ ' to add poisson noise and the JPEG compression ratio is 0.1 .

\section{Color space}

According to the analysis results from $[8,9,11]$, seven different color spaces are selected for our experiment: grayscale space, RGB space, CIELab space, YCbCr space, HSV space, LSLM space, and CIEXYZ space. Therefore, we evaluate the performance of face recognition system by representing face image in nine color components:

- Grayscale: computed from the three components of RGB color space by using the equation: grayscale $=0.299 R+$ $0.587 G+0.114 B$

- Red, Green, and Blue components from RGB color space;

- L (LAB_L) component from CIELab color space: The CIELab color space describes mathematically all perceivable colors in the three dimensions L for lightness and a and $\mathrm{b}$ for the color opponents green?red and blue?yellow;

- Y component from YCbCr color space: The YCbCr color space is widely used for digital video. In this format, luminance information is stored as a single component (Y), and chrominance information is stored as two color-difference components $(\mathrm{Cb}$ and $\mathrm{Cr})$. 
- V component from HSV color space: HSV (Hue, Saturation, Value) is one of the alternative representations of the RGB color space. The HSV representation models the way paints of different colors mix together, with the saturation dimension resembling various shades of brightly colored paint, and the value dimension resembling the mixture of those paints with varying amounts of black or white paint;

- L (LSLM_L) component from LSLM color space: LSLM is a color space represented by the response of the three types of cones of the human eye, named for their responsively (sensitivity) peaks at long, medium, and short wavelengths;

- Z component from CIEXYZ color space: X, Y and Z are extrapolations of RGB created mathematically to avoid negative numbers and are called Tristimulus values. Y means luminance, $\mathrm{Z}$ is somewhat equal to blue, and $\mathrm{X}$ is a mix of cone response curves chosen to be orthogonal to luminance and non-negative.

The color space transformations have been done in Matlab R2016a by using default scripts.

\section{Face recognition system}

The open source face recognition system used in this paper is 'The $\mathrm{PhD}$ (Pretty helpful Development functions) for face recognition toolbox' [19], which is a collection of Matlab functions and scripts for face recognition. The toolbox was produced as a byproduct of Štruc's [19] research work and is freely available for download. The feature extraction algorithms is Gabor Filtering (GF) + Kernel Fisher Analysis (KFA). In this feature extraction algorithm, a bank of complex Gabor filters defined in the spatial and frequency domains will be constructed first. Then, the algorithm computes the magnitude responses of an face image filtered with a filter bank of complex Gabor filters. The magnitude responses of the filtering operations are normalized after downscaling using zero-mean and unit variance normalization. After that they are converted as the feature vector. Before we use the feature vector to perform face recognition, a KFA is applied to it. The KFA method first performs nonlinear mapping from the input space to a high-dimensional feature space, and then implements the multi-class Fisher discriminant analysis in the feature space. The significance of the nonlinear mapping is that it increases the discriminating power of the KFA method, which is linear in the feature space but nonlinear in the input space. The analyzed feature vector will be finally used for face recognition.

\section{Methods for the evaluation the performance of face recognition system}

To evaluate the performance of face recognition systems many measures exist [20]. Among all of them we can first consider the histograms of comparison scores. They are obtained from the genuine (comparison between samples from the same subject) and imposter (comparison between samples from different subjects) comparisons for all image samples. In general, high quality biometric samples could generate relatively 'good' genuine comparison scores (in our case, a score closer to 1 the more similar the two face samples), which are well separated from imposter comparison scores [20]. A color component is useful if it can at least give an ordered indication of an eventual performance [20]. Rank-ordered Detection Error Trade-off (DET) character-

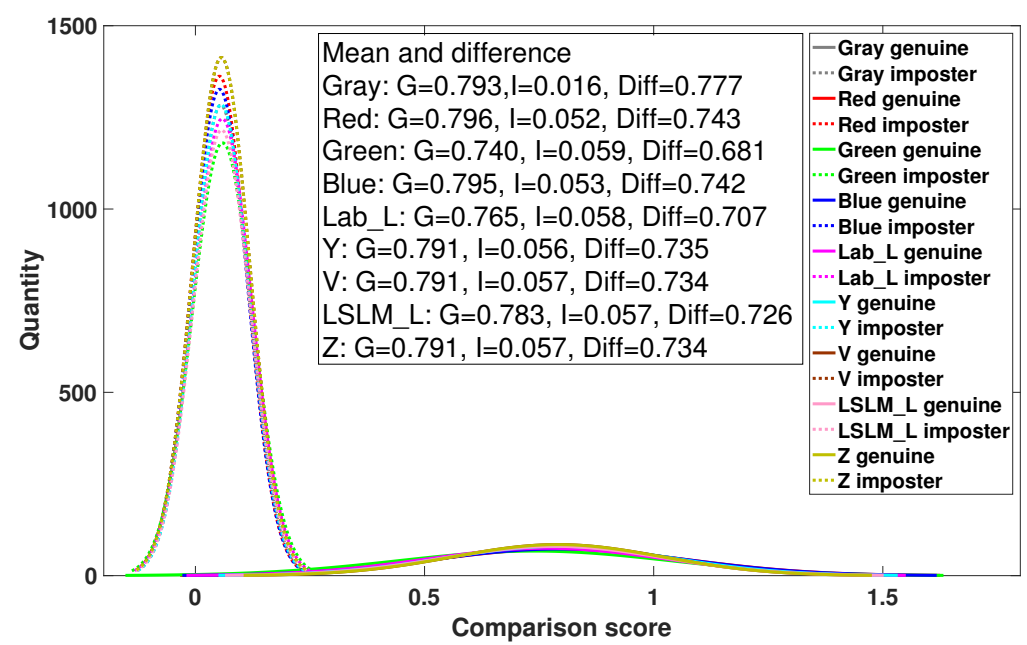

(a) LFC

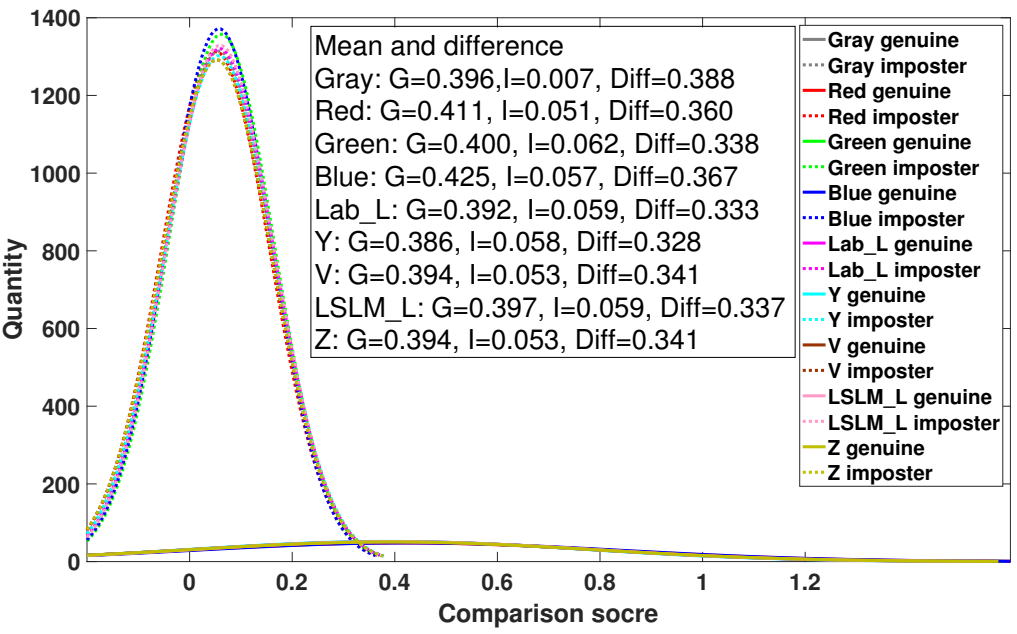

(b) Smartphone

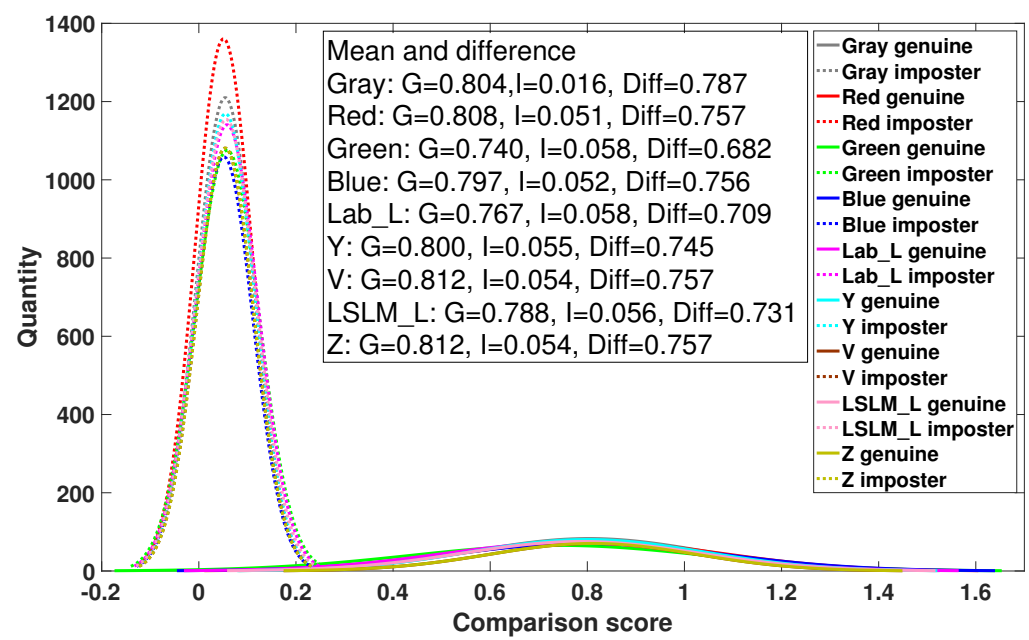

(a) Reflex

Figure 1. Comparison scores and the difference of their mean values from three cameras by using different color space components. 
istics curve is one of the most commonly used and widely understood methods used to evaluate the performance of biometric recognition system. The DET curve used here plots False Match Rate (FMR) versus False None Match Rate (FNMR). Equal Error Rate (EER) (when FMR and FNMR are equal) is another most commonly used method to evaluate the performance of a biometric system. We use it as the third indicator to represent the biometric system performance in this paper.

\section{Experimental results}

\section{Histogram of the comparison scores and the dif- ference of their mean values}

In order to evaluate the performance of face recognition system on degraded face images when taking into account color space components, we first plot the fitted histogram of the comparison scores and the difference of their mean values in Figure 1. The $\mathrm{x}$-axis represents the score and the y-axis represents the quantity of the comparison. The comparisons are between all face images (original and degraded) for each capture device. The reference images are created from each captured sample face image per subject per device. The line plots (continuous lines for genuine comparison and dotted lines for imposter comparison) is the fitted line for the histogram of the comparison score. The mean values and their differences when using different color components to represent the face images are also given in the figures. The $\mathrm{G}$ means the genuine comparison and the I means the imposter comparison. The gray color represents the comparison score from grayscale face images, the red lines represent the red channel from RGB color space, the green lines represent the green channel from RGB color space, the blue lines represent the blue channel from RGB color space, the magenta lines represent the $\mathrm{L}$ channel from CIELab color space, the light blue lines represent the $\mathrm{Y}$ channel from the $\mathrm{YCbCr}$ color space, the brown lines represent the $\mathrm{V}$ channel from HSV color space, the pink lines represent the L channel from the LSLM color space, and the chartreuse lines represent the $\mathrm{Z}$ channel from CIEXYZ color space.

From these three Figures we can see that, when the color space changed there is no big difference between the fitted histogram and the mean of the comparison score. It means that using different components from selected color spaces cannot significantly affect the performance of the face recognition system on degraded face images. However, the biggest differences between the genuine score and the imposter score for three cameras are always from the grayscale face images. It means that grayscale face images have an overall better performance for all three cameras when considering only the $\mu$ values and ignoring the $\sigma$ values.

\section{DET curve}

As mentioned before, we also obtain the DET curve as an indicator to examine the performance of face recognition system by using different color components to represent degraded face images. The DET curves for three cameras are given in Figure 2. The $x$-axis represents the FMR and the y-axis represents the FNMR. If a DET curve is closer to the top-right point, it means that this set of data lead to a higher face recognition performance.

From Figure 2 we can see that, DET curves are overlapping with each other for smartphone. However, for LFC and reflex camera, the better performing color component is $\mathrm{Z}$ channel from

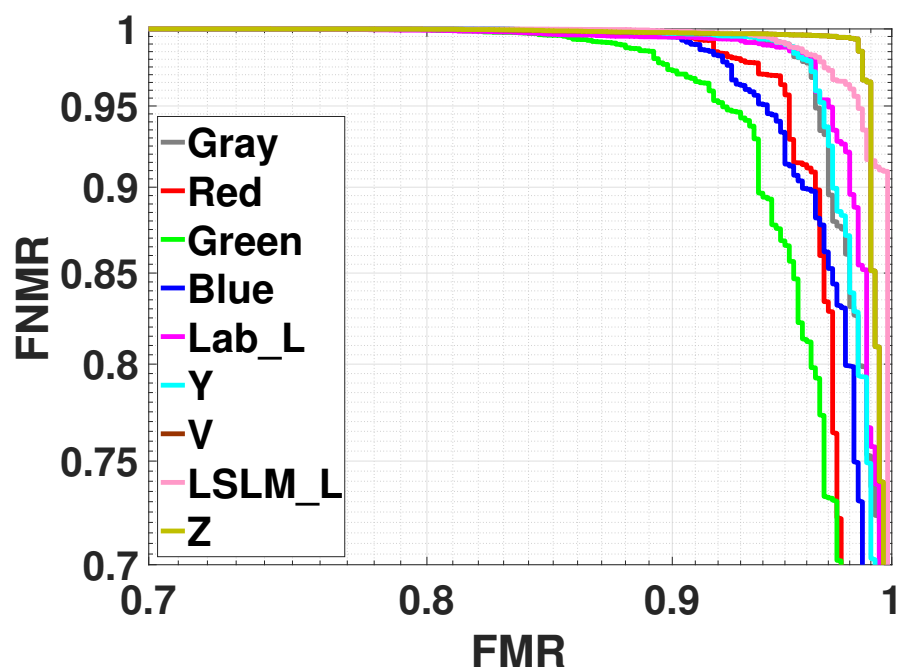

(a) LFC

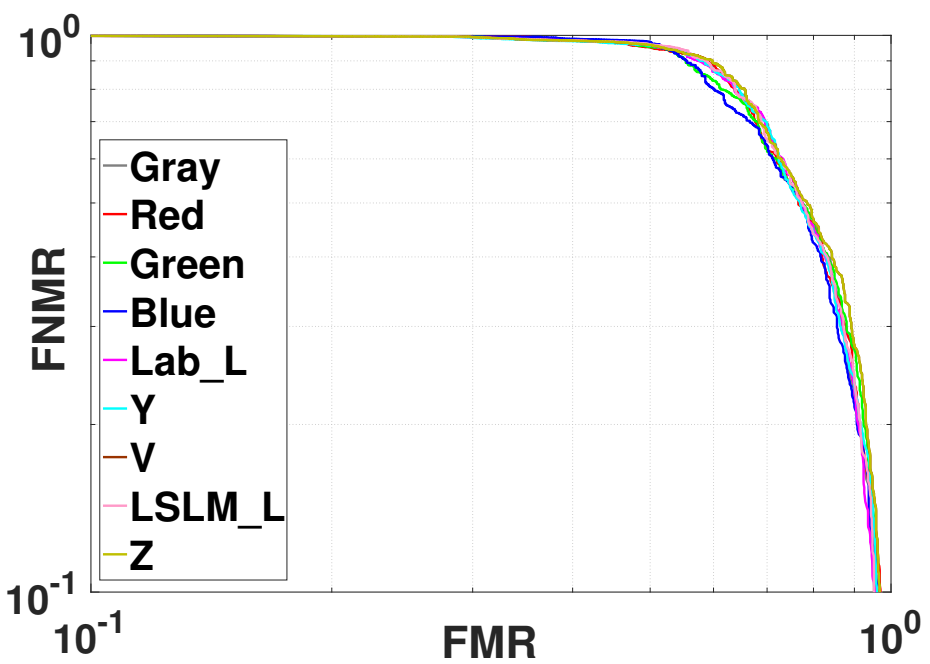

(b) Smartphone

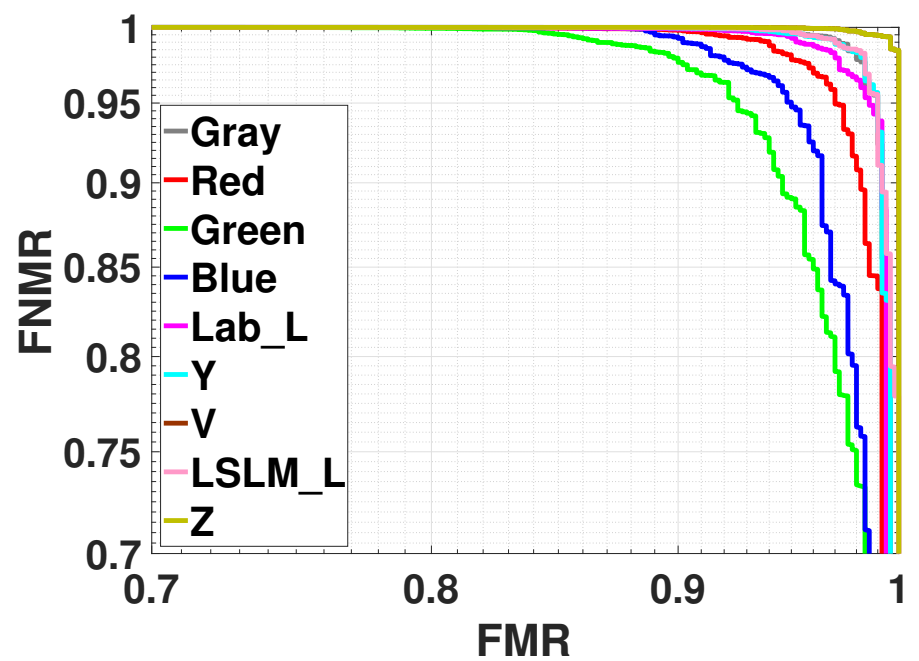

(a) Reflex

Figure 2. DET curves from three cameras by using different color space components. 
Table 1. EER obtained from face recognition by using different color components. Values in red represents the lowest EER for the acquisition devices.

\begin{tabular}{|c|c|c|c|}
\hline $\begin{array}{c}\text { Color } \\
\text { component }\end{array}$ & LFC & Smartphone & Reflex \\
\hline Gray & 0.036 & $\mathbf{0 . 3 0 2}$ & 0.020 \\
\hline Red & 0.048 & 0.317 & 0.034 \\
\hline Green & 0.064 & 0.316 & 0.066 \\
\hline Blue & 0.056 & 0.318 & 0.050 \\
\hline Lab_L & 0.036 & 0.304 & 0.028 \\
\hline Y & 0.036 & $\mathbf{0 . 3 0 2}$ & 0.018 \\
\hline V & $\mathbf{0 . 0 1 4}$ & 0.310 & $\mathbf{0 . 0 0 6}$ \\
\hline LSLM_L & 0.028 & 0.310 & 0.018 \\
\hline $\mathbf{Z}$ & $\mathbf{0 . 0 1 4}$ & 0.310 & $\mathbf{0 . 0 0 6}$ \\
\hline
\end{tabular}

CIEXYZ color space because the chartreuse lines are closer to the top-right point. On the other hand, green channel from RGB color space always gives lower performance because the green lines are furthest from the top-right point compared to the other lines.

\section{EER}

Finally, we use EER as another indicator to illustrate the performance of the face recognition system when using different color component representing degraded face images. The lower EER the better system performance. In Table 1 we can discover similar findings to the DET curves: by using different color components to represent degraded face images, the face recognition performance is slightly affected. For face images taken by smartphone, EERs obtained from gray components and $\mathrm{Y}$ channel from $\mathrm{YCbCr}$ color space are lower than the other components (see values in red color in the third column of Table 1). For face images taken by LFC and reflex camera, the lower EERs are always from $\mathrm{V}$ channel from HSV color space and Z channel from CIEXYZ color space. On the other hand, lower system performance comes from using green channel for LFC and reflex camera because the EERs are higher than the other components. For smartphone, blue channel gives lower system performance. Similar conclusion can be drawn here: there is not a single color component can significantly increase the face recognition performance than the others. However, V channel from HSV color space and $\mathrm{Z}$ channel from CIEXYZ color space give lower EER for two cameras.

\section{Discussion}

As introduced in the previous section, Yoo et al. [9] found out that $\mathrm{YCbCr}$ and $\mathrm{YCg}$ ' Cr' color spaces can provide better performance than the other color spaces. In [10], using the three components red, green, and blue separately from RGB color space could give better recognition performance compared to the greyscale converted image. However, the above mentioned color spaces cannot significantly improve the face recognition performance in our study, which is similar to the findings presented by Hemery et al. [11]. We discovered that different color spaces can influence the system performance depend on the different applications. In our case, it could be due to the acquisition devices used in our study and the special database developed for this work. Since we only consider image-based distortions in this paper, so the face recognition system might be not sensitive to those distor- tion types. On the other hand, we add different distortion to face images that represented by different color spaces, these degraded face images can have different influence on different color spaces.

\section{Conclusions}

In this paper, we investigate how different color space components affect the recognition system performance on degraded face samples images. Nine color components are selected: grayscale; red, green and blue channels from RGB color space; $\mathrm{L}$ channel from CIELab color space; Y channel from $\mathrm{YCbCr}$ color space; V channel from HSV color space; L channel from LSLM color space; and Z channel from CIEXYZ color space. We use three indicators to present system performance: histogram of comparison scores with their mean values and differences, DET curves, and EER. We can conclude from the experimental results that, all selected color components have similar influence to the performance of face recognition system depend on the acquisition devices and the experimental setups. However, depending on different applications, the face recognition performance can be improved by using $\mathrm{Z}$ channel from CIEXYZ color space representing face images taken by LFC and reflex camera.

\section{Acknowledgment}

This research is supported by the Conseil Regional BasseNormandie Grant 14P02048 and Research Council of Norway through project number 221073: HyPerCept - Color and Quality in Higher Dimensions.

\section{References}

[1] Jain, Anil K., and Stan Z. Li. Handbook of face recognition. New York: springer, 2011.

[2] Torres, Luis, Jean-Yves Reutter, and Luis Lorente. "The importance of the color information in face recognition.” Image Processing, 1999. ICIP 99. Proceedings. 1999 International Conference on. Vol. 3. IEEE, 1999.

[3] Choi, Jae Young, Yong Man Ro, and Konstantinos N. Plataniotis. "Color local texture features for color face recognition." IEEE transactions on image processing 21, no. 3 (2012): 1366-1380.

[4] Jones, Creed, and A. Lynn Abbott. "Color face recognition by hypercomplex gabor analysis." In Automatic Face and Gesture Recognition, 2006. FGR 2006. 7th International Conference on, pp. 6-pp. IEEE, 2006.

[5] Wang, Chengzhang, Baocai Yin, Xiaoming Bai, and Yanfeng Sun. "Color face recognition based on 2DPCA." In Pattern Recognition, 2008. ICPR 2008. 19th International Conference on, pp. 1-4. IEEE, 2008.

[6] Yang, Jian, and Chengjun Liu. "A general discriminant model for color face recognition.” In Computer Vision, 2007. ICCV 2007. IEEE 11th International Conference on, pp. 1-6. IEEE, 2007.

[7] Choi, Jae Young, Yong Man Ro, and Konstantinos N. Plataniotis. "Color face recognition for degraded face images." IEEE Transactions on Systems, Man, and Cybernetics, Part B (Cybernetics) 39, no. 5 (2009): 1217-1230.

[8] Yip, Andrew W., and Pawan Sinha. "Contribution of color to face recognition." Perception 31, no. 8 (2002): 995-1003.

[9] Yoo, Seunghwan, Rae-Hong Park, Dong-Gyu Sim, and Nowon-gu Wolgye-dong. "Investigation of Color Spaces for Face Recognition." In MVA, pp. 106-109. 2007.

[10] Bours, Patrick, and Kirsi Helkala. "Face recognition using separate 
layers of the RGB image." In Intelligent Information Hiding and Multimedia Signal Processing, 2008. IIHMSP'08 International Conference on, pp. 1035-1042. IEEE, 2008.

[11] Hemery, Baptiste, Jean-Jacques Schwartzmann, and Christophe Rosenberger. "Study on color spaces for single image enrolment face authentication." In Pattern Recognition (ICPR), 2010 20th International Conference on, pp. 1249-1252. IEEE, 2010.

[12] Jain, Anil K., and Stan Z. Li. Handbook of face recognition. New York: springer, 2011.

[13] Gao, Xiufeng, Stan Z. Li, Rong Liu, and Peiren Zhang. "Standardization of face image sample quality." In International Conference on Biometrics, pp. 242-251. Springer, Berlin, Heidelberg, 2007.

[14] ISO/IEC, "19794-5 biometric data interchange formats - part 5: Face image data," (2011-03-04).

[15] ISO/IEC, "29794-5 information technology - biometric sample quality - part 5: Face image data," (2010).

[16] Liu, Xinwei, Marius Pedersen, Christophe Charrier, and Patrick Bours. "Can no-reference image quality metrics assess visible wavelength iris sample quality?." In IEEE International Conference on Image Processing. 2017.

[17] Liu, Xinwei, Marius Pedersen, and Christophe Charrier. "Imagebased attributes of multi-modality image quality for contactless biometric samples." In Signal Processing and Integrated Networks (SPIN), 2016 3rd International Conference on, pp. 106-111. IEEE, 2016.

[18] Lytro. Lytro, inc. Dans https ://www.lytro.com/about. Visited on $16 / 06 / 2017$

[19] Štruc, V. "The PhD face recognition toolbox: toolbox description and user manual." (2012).

[20] Grother, Patrick, and Elham Tabassi. "Performance of biometric quality measures." IEEE transactions on pattern analysis and machine intelligence 29, no. 4 (2007): 531-543.

\section{Author Biography}

Xinwei Liu obtained his Master degree in Computer Science and Media Technology in 2014 from Gjøvik University College, Norway. He is now approaching to the end of his co-tutelle Ph.D in Biometrics image quality assessment from both Norwegian University of Science and Technology, Gjфvik, Norway, and University of Caen, CNRS, GREYC Lab, Caen, France. His current research interests include image quality assessment, perceptual image quality, biometrics, and biometric image quality.

Christophe Charrier obtained his PhD in Computer Science from the University Jean Monnet (Saint-Etienne) in 1998. From 1998 to 2001, he was a Research Assistant with the Laboratory of Radio Communications and Signal Processing, Laval University, Quebec, QC, Canada. In 2001, he joined the Cherbourg Institute of Technology at Saint-Lo as an assistant professor. In 2008, he joined the GREYC lab and the e-payment and biometrics research unit. In 2008, he was a Visiting Scholar with the Laboratory for Image and Video Engineering, University of Texas, Austin. From 2009 to 2011, he was an Invited Professor with the Computer Department, University of Sherbrooke, Sherbrooke, QC, Canada. His current research interests include digital image and video coding, processing, quality assessment, computational vision, biometrics (evaluation of biometric systems, fingerprint quality assessment...) Since 2016, he is the head of e-payment and biometrics research unit.
Marius Pedersen received his BsC in Computer Engineering in 2006, and MiT in Media Technology in 2007, both from Gjøvik University College, Norway. He completed a PhD program in color imaging in 2011 from the University of Oslo, Norway, sponsored by Océ. He is currently employed as a professor at NTNU Gjovik, Norway. He is also the director of the Norwegian Colour and Visual Computing Laboratory (Colourlab). His work is centered on subjective and objective image quality.

Patrick Bours studied Discrete Mathematics at Eindhoven University of Technology in the Netherlands (MSc 1990, PhD 1994). He worked 10 years at the Netherlands National Communication Security Agency (NLNCSA) as a senior policy member in the area of crypto with a focus on public key crypto and random number generation. He started 7/2005 as a PostDoc at the Norwegian Information Security Laboratory (NISlab) at Gjovik University College. As of 7/2008 he was appointed as Associate Professor at NISlab and specialized in authentication and more specifcally biometrics. His main research interest is in behavioral biometrics include Gait Recognition, Keystroke Dynamics, and Continuous Authentication. From 9/2012 he has a full professor position at NISlab. He was the head of NISlab in the period from 7/2009 till 6/2012. 